\title{
Short and long-term results of endoscopic atraumatic coronary artery off-pump bypass grafting in patients with left anterior descending artery stenosis
}

\author{
Rafik Abusamra ${ }^{1,2}$, Marek Król ${ }^{3}$, Krzysztof Milewski ${ }^{3,4}$, Mateusz Kachel ${ }^{3}$, \\ Loai Abudaqa ${ }^{5}$, Justyna Jankowska-Sanetra ${ }^{3}$, Kamil Derbisz ${ }^{3}$, Krzysztof Sanetra ${ }^{3}$, \\ Anna Sobieszek ${ }^{3}$, Piotr P. Buszman ${ }^{3}$, Wojciech Wojakowski ${ }^{1}$, Paweł E. Buszman ${ }^{1,3}$, \\ Andrzej Bochenek ${ }^{1,3}$, Marek Cisowski ${ }^{1,3}$ \\ ${ }^{1}$ Medical University of Silesia, Katowice, Poland \\ ${ }^{2} \mathrm{Al}$ Zahra Hospital, Sharjah, United Arab Emirates \\ ${ }^{3}$ Center for Cardiovascular Research and Development, American Heart of Poland, Katowice, Poland \\ ${ }^{4}$ The Jerzy Kukuczka Academy of Physical Education, Katowice, Poland \\ ${ }^{5} \mathrm{Al}$ Qassimi Hospital, Sharjah, United Arab Emirates
}

\begin{abstract}
Background: To perform a retrospective analysis of patients who underwent endoscopic atraumatic coronary artery off-pump bypass grafting (EACAB) in a single center over a period of 11 years.

Methods: Data was acquired from the hospital registry and patient medical records. In order to determine changes in clinical profile, patients were subdivided into three groups regarding year of surgery: 1998-2002 (group 1), 2003-2005 (group 2), 2006-2009 (group 3). In-hospital analysis up to 30 days and long-term observation were conducted.

Results: The study cohort consisted of 714 patients (581 male). Procedural success accounted for 99\% of all patients. No mortality was observed up to 30 days. Complications in the early period included pleural effusion (7.6\%), cardiac arrhythmias (3.6\%), bleeding related revision (2.7\%) and wound infection (1.6\%). Mean follow-up was 6 years (2132 \pm 1313 days; median: 1918.5). Nineteen (2.7\%) patients died, of which $52.6 \%$ (10 patients) were due to heart related conditions. Overall frequency of major adverse cerebral and cardiovascular events (MACCE) was 10.8\% (77 patients). The Kaplan-Meyer analysis defined survival rate and event-free survival in long-term observation of $96.1 \%$ and 85.3\%, respectively. Ejection fraction $(E F)<50 \%$ was the only independent factor of mortality (OR: 3.35). Regarding cumulative MACCE, older age (OR: 1.72), lower EF (OR: 3.03), the history of percutaneous coronary intervention (OR: 2.13) and higher New York Heart Association class (OR: 2.63) influenced the incidence rate.

Conclusions: The presented short and very long-term results confirm that EACAB is an efficient alternative for patients requiring revascularization of the left anterior descending artery. The elimination of cardiopulmonary bypass significantly reduces the number of complications. (Cardiol J 2021; 28, 1 : 86-94)

Key words: endoscopic atraumatic coronary artery off-pump bypass grafting (EACAB), minimally invasive surgery, left anterior descending coronary artery stenosis, minimally invasive direct coronary artery bypass grafting (MIDCAB)
\end{abstract}

Address for correspondence: Rafik AbuSamra, MD, PhD, Al Zahra Hospital, Sharjah, United Arab Emirates, tel: +971 6561 9999, e-mail: drrabusamra@yahoo.com

Received: 10.10.2018 Accepted: 8.01.2019

This article is available in open access under Creative Common Attribution-Non-Commercial-No Derivatives 4.0 International (CC BY-NC-ND 4.0) license, allowing to download articles and share them with others as long as they credit the authors and the publisher, but without permission to change them in any way or use them commercially. 


\section{Introduction}

Percutaneous coronary intervention (PCI) and coronary artery bypass grafting (CABG) are widely used revascularization techniques [1]. In a selected group of patients, minimally invasive direct coronary artery bypass grafting (MIDCAB) may be used as an alternative treatment. MIDCAB comprises high efficacy of surgical revascularization combined with a minimal traumatic approach due to the use of anterolateral thoracotomy or endoscopy (endoscopic atraumatic coronary artery bypass grafting [EACAB]) for left internal mammary artery (LITA) harvest and anterolateral thoracotomy for suturing the anastomosis. It results in the avoidance of sternotomy, reduction of wound diameter, improved healing and faster recovery $[2,3]$. Current guidelines for the use of MIDCAB are limited. The procedure is most widely applied in the single vessel disease of the left anterior descending coronary artery (LAD), as a hybrid procedure (along with stenting of other vessels in patients treated for multi-vessel disease), in patients unable to undergo the complete $\mathrm{CABG}$ revascularization and as a palliative therapy in patients with in-stent restenosis $[3,4]$. Studies have proven the efficacy and safety of the MIDCAB procedure in comparison to both CABG and PCI [5-8]. It is estimated that MIDCAB may be indicated in $2-8 \%$ of patients primarily qualified for CABG. This highlights the need for further investigation in order to increase usage of this method. Most available studies addressing MIDCAB lack long-term observation conducted on a large study cohort. Therefore, a retrospective analysis was performed on patients who underwent the procedure in the documented clinic over a period of 11 years. The aim was to collect for the longest possible follow-up and to determine changes in a surgically treated population whose characteristics were revealed over time.

\section{Methods}

\section{Study population}

The study cohort consisted of patients who underwent MIDCAB (EACAB) surgery in First Cardiac Surgery Clinic of Medical University of Silesia from April 1998 through December 2009. To determine the changes in clinical profiles, patients were subdivided into three groups depending on their year of surgery: 1998-2002 (group 1), 2003-2005 (group 2), 2006-2009 (group 3). Retrospective analysis was performed with the use of data acquired from hospital registry and patient medical records. Pre-admission data, such as age, gender, Canadian Cardiovascular Society (CCS) class, New York Heart Association (NYHA) class, body mass index and others were used to calculate the additive EuroSCORE (ES) and logistic EuroSCORE (LES). Arrhythmias and conduction disorders were diagnosed on the basis of an electrocardiogram (ECG).

\section{The surgical technique}

General anesthesia was induced. The doublelumen endotracheal tube was introduced to ensure single lung ventilation throughout the procedure. The harmonic blade and endoscopic manipulators were used for the LITA harvest. When the harvest was completed, heparin was given in a dose adequate to the patient's weight. The incision was made in the fourth or fifth intercostal space and LITA-LAD anastomosis was sutured in a standard technique on the beating heart, with the use of cardiac stabilizer. The protamine sulphate was administered after completion of the anastomosis.

\section{The primary and secondary endpoints}

The primary endpoint was major adverse cerebral and cardio-vascular event (MACCE) incidence throughout the observation period. The MACCE was defined as: death, myocardial infarction (MI), repeat cardiac revascularization (both surgical or percutaneous), stroke or transient ischemic attack (TIA). The secondary endpoint consisted of post-operative renal insufficiency, hemorrhage and prolonged hospitalization. The MI was diagnosed in accordance to the American College of Cardiology/American Heart Association (ACC/AHA) guidelines, based on ECG changes, the levels of cardiac enzymes and the evaluation of the cardiac contractility with transthoracic echocardiography (TTE) or transesophageal echocardiography (TEE). Neurological disorders were diagnosed with the use of imaging modalities (computed tomography scan) and physical examination conducted by a qualified neurologist.

\section{Peri-procedural observation}

In-hospital analysis consisted of peri-procedural parameters assessment, observation focused on both primary and secondary endpoints, as well as the incidence of other possible complications at the time of surgery and throughout the subsequent 30 days.

\section{Long-term observation}

Long-term observation encompassed an evaluation of post hospital mortality and occurrence 
of MACCE. Patients with incomplete in-hospital data were excluded from analysis. Follow up was gathered via telephone survey by qualified study coordinators. When telephone contact was not possible, mail was sent to the patients' address with a request to mail back a questionnaire to be filled in and, if available, printed history cards from other hospitalizations. Information regarding mortality was confirmed owing to access to the Polish central population registry database called PESEL.

\section{Statistical analysis}

Statistical analysis was performed using Statistica 7.1 software by a qualified statistician. Differences between groups were assessed with the Student t-test and variation analysis for quantitative variables and with an $\chi^{2}$ or the Fisher test for qualitative variables. The cumulative survival rate was described using the Kaplan-Meier estimator plot. Factors influencing MACCE incidence were typed using log-rank analysis. For the proper interpretation of differences and correlations, statistical significance parameter was $\mathrm{p}<0.05$.

\section{Results}

\section{Study population}

The study cohort consisted of 714 patients (581 male, 133 female). There were 238 patients in group 1 (33.3\%), 229 patients in group $2(32.1 \%)$ and 247 patients in group 3 (34.6\%). Detailed study cohort characterizations are shown in Table 1. The majority of patients presented with good left ventricular ejection fraction (LVEF) $>50 \%$ within average of $55 \%$, had mild angina (CCS II: $86.1 \%$ ) and preserved heart function (NYHA I: $91.8 \%$ ). Therefore, they remained in a group of low surgical risk, which was reflected both in additive and logistic EuroSCORE (values of ES $<3$ points in $68.7 \%$ of patients and values of LES: $2.0 \pm 1.9 \%$ ).

\section{Peri-procedural observation}

Ninety-nine percent of patients successfully underwent MIDCAB (EACAB) procedure. In $1 \%$ of cases ( 7 procedures) the conversion to OPCAB (4 patients) or CABG (3 patients) were required. Only $9.5 \%$ of surgeries were urgent (due to exacerbation of angina symptoms or critical LAD stenosis), the remaining were planned. All patients underwent LAD grafting. Most of them (99.5\%) received left internal thoracic artery graft. In $0.5 \%$ of cases the use of LITA was impossible due to damage made to the vessel. Incomplete revascularization (patients requiring further percutaneous
Table 1. Study cohort characterization.

\begin{tabular}{|c|c|}
\hline Variable & Value \\
\hline Age [years] & $\begin{array}{c}57.9 \pm 9.7 \\
(57.0 ; 51-65)\end{array}$ \\
\hline Body weight [kg] & $\begin{array}{c}74.6 \pm 16.1(75.5 \\
64.5-85)\end{array}$ \\
\hline Height $[\mathrm{m}]$ & $\begin{array}{c}1.70 \pm 0.08 \\
(1.71 ; 1.64-1.76)\end{array}$ \\
\hline BMI $\left[\mathrm{kg} / \mathrm{m}^{2}\right]-$ Mean & $\begin{array}{c}27.6 \pm 3.8 \\
(27.6 ; 25.5-29.4)\end{array}$ \\
\hline $\mathrm{BMI} \geq 25 \mathrm{~kg} / \mathrm{m}^{2}$ & $75.2 \%$ \\
\hline CCS class - Mean & $\begin{array}{l}2.0 \pm 0.37 \\
(2.0 ; 2-2)\end{array}$ \\
\hline 1 & $6.8 \%$ \\
\hline 2 & $86.1 \%$ \\
\hline $3 / 4$ & $7.1 \%$ \\
\hline NYHA class - Mean & $\begin{array}{l}1.1 \pm 0.27 \\
(1.0 ; 1-1)\end{array}$ \\
\hline I & $91.8 \%$ \\
\hline II & $8.2 \%$ \\
\hline III/IV & - \\
\hline Arterial hypertension & $51.5 \%$ \\
\hline Diabetes & $13.9 \%$ \\
\hline MI in history & $39.8 \%$ \\
\hline $\mathrm{PCl}$ in history & $24.6 \%$ \\
\hline Dyslipidemia & $57.4 \%$ \\
\hline Nicotinism & $79 \%$ \\
\hline Renal insufficiency & $1 \%$ \\
\hline Peptic ulcer disease in history & $1.5 \%$ \\
\hline Peripheral artery disease & $2 \%$ \\
\hline Stroke/TIA in history & $0.8 \%$ \\
\hline Thyroid disease & $1.8 \%$ \\
\hline COPD & $2.8 \%$ \\
\hline $\begin{array}{l}\text { EuroSCORE } \\
\text { [points] - Mean }\end{array}$ & $\begin{array}{c}1.88 \pm 1.87 \\
(2.0 ; 0-3)\end{array}$ \\
\hline $0-2$ & $68.7 \%$ \\
\hline $3-5$ & $28.6 \%$ \\
\hline$>5$ & $2.7 \%$ \\
\hline Logistic EuroSCORE [\%] & $\begin{array}{c}2.0 \pm 1.9 \\
(1.33 ; 0.88-2.36)\end{array}$ \\
\hline $\begin{array}{l}\text { Ejection fraction } \\
{[\%] \text { - Mean }}\end{array}$ & $\begin{array}{c}54.7 \pm 7.4 \\
(55 ; 50-60)\end{array}$ \\
\hline$>50 \%$ & $74.2 \%$ \\
\hline $30-50 \%$ & $25.6 \%$ \\
\hline$<30 \%$ & $0.2 \%$ \\
\hline \multicolumn{2}{|l|}{ Valvular disease } \\
\hline $\mathrm{MVI}+/++$ & $3 \%$ \\
\hline $\mathrm{TVI}+/++$ & $0.3 \%$ \\
\hline
\end{tabular}

$\mathrm{BMI}$ - body mass index; CCS - Canadian Cardiovascular Society; COPD - chronic obstructive pulmonary disease; $\mathrm{MI}$ - myocardial infarction; MVI - mitral valve insufficiency; NYHA - New York Heart Association; $\mathrm{PCl}$ - percutaneous coronary intervention; TIA — transient ischemic attack; TVI — tricuspid valve insufficiency 
treatment) was observed in $1 \%$ of patients. The complete post-op drainage amounted $593.2 \pm$ $\pm 509.5 \mathrm{~mL}$ on average (median [Mdn]: 425 , interquartile range [IQR]: 260-720).

No deaths were observed for up to 30 days after the surgery. Complications in the early postoperative period were rare and included pleural effusion $(7.0 \%)$, cardiac arrhythmias (3.6\%), bleeding related revision $(2.7 \%)$ and wound infection $(1.6 \%)$. Transfusion of red cell concentrate, frozen plasma and platelet concentrate was required in $5 \%, 4,7 \%$ and $0.6 \%$ of patients, respectively. The average stay in the post-operative ward was $2 \pm 0.7$ days (Mdn: 2 , IQR: 2-2) with an overall hospital stay of $7 \pm$ \pm 1.2 days (Mdn: 7, IQR: $6-8$ ). Detailed information about observed complications is listed in Table 2 .

\section{Clinical profile changes}

Similar to the trend observed in cardiac surgery in general, patients undergoing the procedure in subsequent years were increasingly older and suffered from more concomitant diseases (Table 3 ). The reported difference in age was 3 and 6 years between groups 1 vs. 2 and 1 vs. 3 , respectively $(\mathrm{p}<0.01)$. Other statistically significant differences included body weight (with peak value reported in group $2 ; \mathrm{p}<0.01$ ), presence of arterial hypertension $(\mathrm{p}<0.01)$, diabetes $(\mathrm{p}<0.01)$, peptic ulcer disease $(\mathrm{p}<0.01)$, past episodes of MI $(\mathrm{p}=0.03)$, history of stroke/TIA $(\mathrm{p}=0.02)$, history of PCI $(\mathrm{p}<0.01)$ and renal insufficiency $(p=0.03)$. Patients qualified for the surgery were also more frequently diagnosed as having mitral $(p<0.01)$ or tricuspid valve regurgitation $(p<0.04)$ concomitantly. What was understandable, all of this translated into a rise in EuroSCORE value, both additive (from $1.11 \pm 1.12$ in group 1 to $2.11 \pm$ \pm 1.95 in group 3$)$ and logistic (1.12 \pm 0.75 to $2.26 \pm 224)$. Despite visible changes, this difference appeared to be statistically insignificant, reaching $\mathrm{p}=0.06$. Interestingly, no variations were reported in the LVEF. In following years, the median LVEF was stable and amounted to $55 \%(\mathrm{p}=0.13)$.

The urgency of procedures evolved over time. In group 1 all patients were admitted for planned surgery, whereas in group 2 and 3 the amount diminished to $95.8 \%$ and $78.5 \%$, respectively, giving rise to ones conducted due to urgent causes. What is more, the analysis of in-hospital complications showed that MI $(\mathrm{p}<0.01)$ and sudden cardiac arrest $(\mathrm{p}=0.01)$ were more likely to happen in early years. No other variations were reported in frequency of complications, as well as in hospital stay duration (Table 4).
Table 2. Periprocedural complications (up to 30 days).

\begin{tabular}{lc}
\hline Type of complication & Frequency \\
\hline Death & $0 \%$ \\
Left ventricular insufficiency & $0.1 \%$ \\
Sudden cardiac arrest & $0.6 \%$ \\
Myocardial infarction & $1.5 \%$ \\
IABP & $0.4 \%$ \\
Inotropic support: & \\
Aggregate & $1.3 \%$ \\
Epinephrine & $0.3 \%$ \\
Norepinephrine & $1.0 \%$ \\
Dopamine & $1.0 \%$ \\
Arrhythmias (AF/SVT) & $3.6 \%$ \\
Conduction disorders & $0 \%$ \\
Stroke/TIA & $0.2 \%$ \\
Acute kidney injury & $0 \%$ \\
Acute lower limb ischemia & $0 \%$ \\
Prolonged ventilation (> $48 \mathrm{~h})$ & $0.1 \%$ \\
Reintubation & $0.1 \%$ \\
Gastrointestinal bleeding & $0.1 \%$ \\
Delirium & $0.6 \%$ \\
Multi organ failure & $0.1 \%$ \\
Systemic infection & $0 \%$ \\
Wound infection & $1.6 \%$ \\
Pleural effusion & $7.0 \%$ \\
Chest revision surgery & $2.7 \%$ \\
\hline
\end{tabular}

$\mathrm{AF}$ - atrial fibrillation; IABP - intra-aortic balloon pump; SVT supraventricular tachycardia; TIA — transient ischemic attack

\section{Long-term observation}

The average follow-up time was almost 6 years (2132 \pm 1313 days; Mdn: 1918.5) with the longest spanning up to 13 years (4661 days). Nineteen (2.7\%) patients died during observation period, of whom $52.6 \%$ (10 patients) due to heart related conditions. Overall frequency of MACCE was $10.8 \%$ (77 patients). The most frequent complication was the need for repeat revascularization in $50(7 \%)$ patients with angioplasty of LIMA-LAD graft being responsible for $38 \%$ of cases (19/50). It was followed by MI in $2.4 \%$ (17 patients) of cases and stroke or TIA in 1\% (7) of patients. The Kaplan-Meyer analysis defined survival rate and event-free survival in longterm observation of $96.1 \%$ and $85.3 \%$, respectively (Figs. 1,2). Detailed analysis revealed that LVEF $<50 \%$ was the only independent factor of mortality (odds ratio [OR]: 3.35). Age $>57$, history of PCI and higher NYHA class were on 
Table 3. Clinical profile changes.

\begin{tabular}{|c|c|c|c|c|}
\hline Variable & $\begin{array}{c}\text { Group } 1 \\
(1998-2002)\end{array}$ & $\begin{array}{c}\text { Group 2 } \\
(2003-2005)\end{array}$ & $\begin{array}{c}\text { Group 3 } \\
(2006-2009)\end{array}$ & $\mathbf{P}$ \\
\hline Male sex & $84.9 \%$ & $74.5 \%$ & $79.8 \%$ & 0.15 \\
\hline Age [years] & $55.1 \pm 9.6$ & $58.5 \pm 9.6$ & $59.9 \pm 9.2$ & 0.001 \\
\hline Body weight [kg] & $71.9 \pm 16.4$ & $81.6 \pm 14.1$ & $79.8 \pm 13.3$ & 0.001 \\
\hline Height $[\mathrm{m}]$ & $1.72 \pm 8.2$ & $1.71 \pm 8.4$ & $1.7 \pm 7.4$ & 0.68 \\
\hline BMI $\left[\mathrm{kg} / \mathrm{m}^{2}\right]$ & $25.1 \pm 4.1$ & $27.8 \pm 3.6$ & $27.5 \pm 4.1$ & 0.51 \\
\hline$\geq 25 \mathrm{~kg} / \mathrm{m}^{2}$ & $58 \%$ & $79 \%$ & $78 \%$ & 0.42 \\
\hline CCS class: & $1.98 \pm 0.33$ & $2.01 \pm 0.41$ & $2.02 \pm 0.39$ & 0.59 \\
\hline 1 & $6.5 \%$ & $7.5 \%$ & $6.5 \%$ & 0.57 \\
\hline 2 & $88.8 \%$ & $84.1 \%$ & $85 \%$ & \\
\hline $3 / 4$ & $4.7 \%$ & $8.4 \%$ & $8.5 \%$ & \\
\hline NYHA class: & $1.04 \pm 0.19$ & $1.13 \pm 0.34$ & $1.08 \pm 0.28$ & 0.11 \\
\hline 1 & $96.2 \%$ & $86.9 \%$ & $91.6 \%$ & 0.05 \\
\hline II & $3.8 \%$ & $13.1 \%$ & $8.4 \%$ & \\
\hline EuroSCORE [points]: & $1.11 \pm 1.12$ & $1.51 \pm 1.69$ & $2.11 \pm 1.95$ & 0.06 \\
\hline $0-2$ & $72.5 \%$ & $68.7 \%$ & $64.2 \%$ & 0.03 \\
\hline $3-5$ & $26 \%$ & $28.5 \%$ & $32.5 \%$ & \\
\hline$>5$ & $1.5 \%$ & $2.8 \%$ & $3.3 \%$ & \\
\hline Logistic EuroSCORE [\%] & $1.12 \pm 0.75$ & $1.53 \pm 0.89$ & $2.26 \pm 2.24$ & 0.06 \\
\hline Ejection fraction [\%]: & $55.8 \pm 8.0$ & $54.2 \pm 6.6$ & $54.2 \pm 7.2$ & 0.13 \\
\hline$>50 \%$ & $76.2 \%$ & $73.8 \%$ & $75.1 \%$ & 0.61 \\
\hline $30-50 \%$ & $23.7 \%$ & $26 \%$ & $24.7 \%$ & \\
\hline$<30 \%$ & $0.1 \%$ & $0.2 \%$ & $0.2 \%$ & \\
\hline \multicolumn{5}{|l|}{ Valvular disease: } \\
\hline $\mathrm{MVI}+/++$ & 0 & $4.2 \%$ & $5.8 \%$ & 0.001 \\
\hline $\mathrm{TVI}+/++$ & 0 & 0 & $1.3 \%$ & 0.04 \\
\hline Arterial hypertension & $45.2 \%$ & $49.5 \%$ & $69 \%$ & 0.001 \\
\hline Diabetes & $9.5 \%$ & $14 \%$ & $18.9 \%$ & 0.005 \\
\hline MI in history & $35.3 \%$ & $38.9 \%$ & $44.9 \%$ & 0.03 \\
\hline $\mathrm{PCl}$ in history & $11 \%$ & $29.7 \%$ & $33.1 \%$ & 0.001 \\
\hline Dyslipidemia & $53 \%$ & $62 \%$ & $57.4 \%$ & 0.32 \\
\hline Nicotinism & $75 \%$ & $80 \%$ & $78 \%$ & 0.56 \\
\hline Renal insufficiency & $0.4 \%$ & 0 & $2.4 \%$ & 0.03 \\
\hline Peptic ulcer disease in history & 0 & $1.4 \%$ & $3.4 \%$ & 0.004 \\
\hline Peripheral artery disease & $2.2 \%$ & 0 & $3.8 \%$ & 0.24 \\
\hline Stroke/TIA in history & 0 & $0.4 \%$ & $1.9 \%$ & 0.02 \\
\hline Thyroid disease & $0.8 \%$ & $1.4 \%$ & $3.3 \%$ & 0.05 \\
\hline $\begin{array}{l}\text { Chronic obstructive pulmonary } \\
\text { disease }\end{array}$ & $1.3 \%$ & $4.2 \%$ & $2.8 \%$ & 0.21 \\
\hline
\end{tabular}

BMI - body mass index; CCS - Canadian Cardiovascular Society; MI - myocardial infarction; MVI - mitral valve insufficiency; NYHA - New York Heart Association; $\mathrm{PCl}$ - percutaneous coronary intervention; TIA - transient ischemic attack; TVI — tricuspid valve insufficiency

the edge of statistical significance. Regarding cumulative MACCE, older age (OR: 1.72), lower LVEF (OR: 3.03), the history of PCI (OR: 2.13) and higher NYHA class (OR: 2.63) influenced the incidence rate. The risk of MI rose with the presence of lower LVEF (OR: 3.56) and incom- plete revascularization (OR: 6.58), whereas risk of stroke/TIA was greater when the patient was over 57 years old (OR: 6.30). Other important, but not statistically significant, factors were the presence of arterial hypertension and male sex. Detailed information is shown in Table 5. 
Table 4. Periprocedural complications (up to 30 days) with subgroup analysis.

\begin{tabular}{|c|c|c|c|c|c|}
\hline Type of complication & $\begin{array}{c}\text { Group } 1 \\
(1998-2002)\end{array}$ & $\begin{array}{c}\text { Group } 2 \\
(2003-2005)\end{array}$ & $\begin{array}{c}\text { Group } 3 \\
(2006-2009)\end{array}$ & $\mathbf{P}^{*}$ & $\begin{array}{c}\text { Mean } \\
\text { aggregate }\end{array}$ \\
\hline Death & 0 & 0 & 0 & - & $0 \%$ \\
\hline Left ventricular insufficiency & $0.4 \%$ & 0 & 0 & 0.21 & $0.1 \%$ \\
\hline Sudden cardiac arrest & $1.7 \%$ & 0 & 0 & 0.01 & $0.6 \%$ \\
\hline Myocardial infarction & $4.2 \%$ & $0.4 \%$ & 0 & 0.001 & $1.5 \%$ \\
\hline IABP & $0.4 \%$ & 0 & 0 & 0.47 & $0.4 \%$ \\
\hline \multicolumn{6}{|l|}{ Inotropic support: } \\
\hline $1.5 \%$ & $0.8 \%$ & $0.6 \%$ & 0.11 & 0.11 & $1.3 \%$ \\
\hline $0.4 \%$ & 0 & 0 & 0.21 & 0.21 & $0.3 \%$ \\
\hline $1.8 \%$ & 0 & $0.8 \%$ & 0.14 & 0.14 & $1.0 \%$ \\
\hline $1.5 \%$ & $1 \%$ & 0 & 0.21 & 0.21 & $1.0 \%$ \\
\hline Arrhythmias (AF/SVT) & $4.2 \%$ & $3.1 \%$ & $3.6 \%$ & 0.74 & $3.6 \%$ \\
\hline Conduction disorders & 0 & 0 & 0 & - & $0 \%$ \\
\hline Stroke/TIA & 0 & 0 & $0.8 \%$ & 0.09 & $0.2 \%$ \\
\hline Acute kidney injury & 0 & 0 & 0 & - & $0 \%$ \\
\hline Acute lower limb ischemia & 0 & 0 & 0 & - & $0 \%$ \\
\hline Prolonged ventilation ( $>48 \mathrm{~h}$ ) & $0.4 \%$ & 0 & 0 & 0.21 & $0.1 \%$ \\
\hline Reintubation & 0 & 0 & $0.4 \%$ & 0.21 & $0.1 \%$ \\
\hline Gastrointestinal bleeding & $0.4 \%$ & 0 & 0 & 0.21 & $0.1 \%$ \\
\hline Delirium & $0.4 \%$ & $0.9 \%$ & $0.4 \%$ & 0.97 & $0.6 \%$ \\
\hline Multi organ failure & 0 & 0 & $0.4 \%$ & 0.21 & $0.1 \%$ \\
\hline Systemic infection & 0 & 0 & 0 & - & $0 \%$ \\
\hline Wound infection & $1.7 \%$ & $1.7 \%$ & $1.2 \%$ & 0.31 & $1.6 \%$ \\
\hline Pleural effusion & $5.8 \%$ & $7.5 \%$ & $6 \%$ & 0.94 & $7.0 \%$ \\
\hline Chest revision surgery & $3.4 \%$ & $3.5 \%$ & $1.2 \%$ & 0.14 & $2.7 \%$ \\
\hline
\end{tabular}

*Statistical significance defined as $\mathrm{p}<0.05 ; \mathrm{AF}$ - atrial fibrillation; IABP — intra-aortic balloon pump; SVT — supraventricular tachycardia TIA - transient ischemic attack

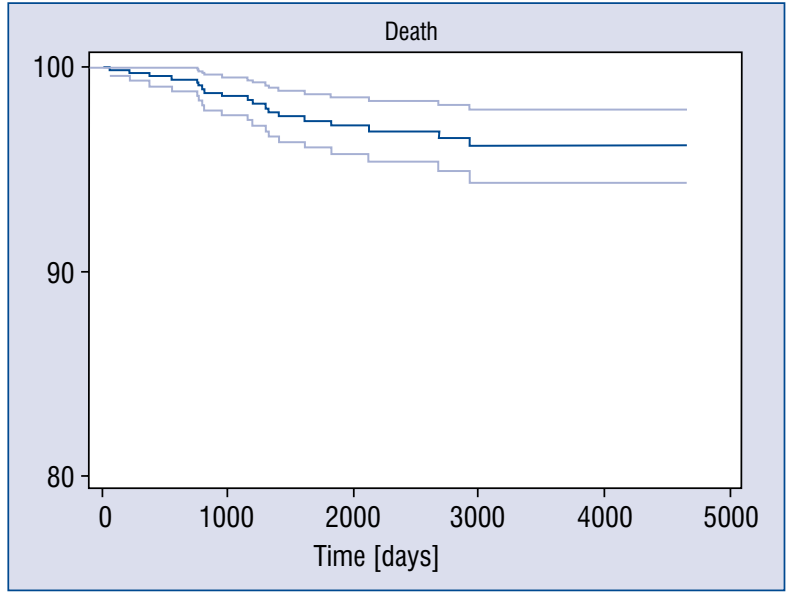

Figure 1. Kaplan-Mayer survival rate of $96.1 \%$.

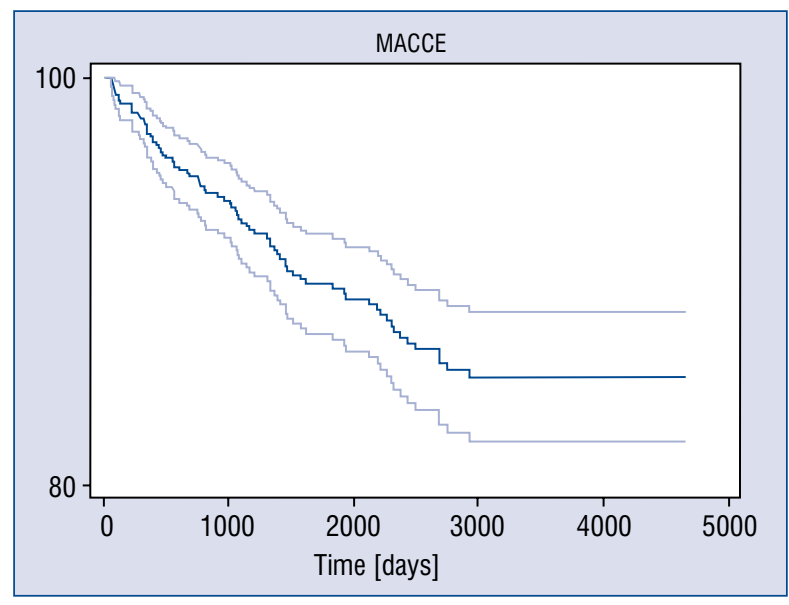

Figure 2. Major adverse cerebral and cardiovascular events (MACCE) - free survival of $85.3 \%$. 
Table 5. Major adverse cerebral and cardiovascular events (MACCE) risk factors analysis in very long-term observation.

\begin{tabular}{|c|c|c|c|c|c|}
\hline & MACCE & Death & MI & RE-PCI & Stroke/TIA \\
\hline Male sex & $\begin{array}{c}1.17(0.62-2.16) \\
p=0.72\end{array}$ & $\begin{array}{l}0(0-1.13) \\
p=0.15\end{array}$ & $\begin{array}{c}1.35(0.37-4.55) \\
p=0.83\end{array}$ & $\begin{array}{c}1.42(0.68-2.91) \\
p=0.41\end{array}$ & $\begin{array}{c}3.33(0.58-17.81) \\
p=0.24\end{array}$ \\
\hline Age $>57$ years & $\begin{array}{c}1.72(1.03-2.86) \\
p=0.02\end{array}$ & $\begin{array}{c}2.29(0.88-6.83) \\
p=0.08\end{array}$ & $\begin{array}{c}0.92(0.32-2.62) \\
p=0.94\end{array}$ & $\begin{array}{c}1.47(0.79-2.73) \\
p=0.25\end{array}$ & $\begin{array}{c}6.30(0.75-139) \\
p=0.05\end{array}$ \\
\hline$E F<50 \%$ & $\begin{array}{c}3.03(1.49-6.25) \\
p=0.001\end{array}$ & $\begin{array}{c}3.55(1.03-11.59 \\
p=0.03\end{array}$ & $\begin{array}{c}3.56(1.49-20.6) \\
p=0.005\end{array}$ & $\begin{array}{c}0.49(0.19-1.31) \\
p=0.18\end{array}$ & $\begin{array}{c}3.76(0.47-24.55) \\
p=0.10\end{array}$ \\
\hline Diabetes & $\begin{array}{c}1.45(0.72-2.88) \\
p=0.34\end{array}$ & $\begin{array}{c}1.98(0.53-6.72) \\
p=0.40\end{array}$ & $\begin{array}{c}1.49(0.33-5.70) \\
p=0.79\end{array}$ & $\begin{array}{c}1.32(0.55-3.07) \\
p=0.64\end{array}$ & $\begin{array}{c}1.0(0.98-1.03) \\
p=0.69\end{array}$ \\
\hline Arterial hypertension & $\begin{array}{c}1.05(0.63-1.77) \\
p=0.95\end{array}$ & $\begin{array}{c}1.02(0.36-2.95) \\
p=0.84\end{array}$ & $\begin{array}{c}1.31(0.45-3.86) \\
p=0.76\end{array}$ & $\begin{array}{c}0.79(0.42-1.48) \\
p=0.52\end{array}$ & $\begin{array}{c}4.60(0.52-104) \\
p=0.12\end{array}$ \\
\hline $\begin{array}{l}\text { Incomplete } \\
\text { revascularization }\end{array}$ & $\begin{array}{c}3.30(0.44-19.64) \\
p=0.37\end{array}$ & $\begin{array}{c}0(0-32.03) \\
p=0.44\end{array}$ & $\begin{array}{c}6.58(1-109.1) \\
p=0.05\end{array}$ & $\begin{array}{c}2.18(0.80-3.47) \\
p=0.46\end{array}$ & $\begin{array}{c}0(0-107.67) \\
p=0.79\end{array}$ \\
\hline $\mathrm{PCl}$ in history & $\begin{array}{c}2.13(1.28-3.54) \\
p=0.002\end{array}$ & $\begin{array}{c}2.15(0.79-5.82) \\
p=0.09\end{array}$ & $\begin{array}{c}2.70(0.94-7.80) \\
p=0.06\end{array}$ & $\begin{array}{c}2.11(1.14-3.91) \\
p=0.01\end{array}$ & $\begin{array}{c}1.76(0.31-9.38) \\
p=0.73\end{array}$ \\
\hline MI in history & $\begin{array}{c}0.89(0.52-1.53) \\
p=0.76\end{array}$ & $\begin{array}{c}1.12(0.38-3.25) \\
p=0.98\end{array}$ & $\begin{array}{c}1.83(0.64-5.28) \\
p=0.32\end{array}$ & $\begin{array}{c}0.73(0.37-1.44) \\
p=0.42\end{array}$ & $\begin{array}{c}0.32(0.01-2.79) \\
p=0.49\end{array}$ \\
\hline CCS class $3-4$ vs. $1-2$ & $\begin{array}{c}1.02(0.34-2.83) \\
p=0.97\end{array}$ & $\begin{array}{c}1.0(0.95-1.05) \\
p=0.87\end{array}$ & $\begin{array}{c}1.0(0.95-1.05) \\
p=0.87\end{array}$ & $\begin{array}{c}0.92(0.22-3.28) \\
p=0.86\end{array}$ & $\begin{array}{c}0(0-13.14) \\
p=0.88\end{array}$ \\
\hline NYHA class II vs. I & $\begin{array}{c}2.63(1.15-5.93) \\
p=0.01\end{array}$ & $\begin{array}{c}3.12(0.88-12.33) \\
p=0.08\end{array}$ & $\begin{array}{c}3.12(0.88-12.33) \\
p=0.08\end{array}$ & $\begin{array}{c}1.32(0.38-4.12) \\
p=0.83\end{array}$ & $\begin{array}{c}0(0-13.38) \\
p=0.87\end{array}$ \\
\hline Urgent surgery & $\begin{array}{c}0.47(0.11-1.60) \\
p=0.28\end{array}$ & $\begin{array}{c}1.45(0-6.82) \\
p=0.95\end{array}$ & $\begin{array}{c}0.99(0.96-1.03) \\
p=0.84\end{array}$ & $\begin{array}{l}0(0-2.13) \\
p=0.27\end{array}$ & $\begin{array}{l}0(0-9.78) \\
p=0.96\end{array}$ \\
\hline
\end{tabular}

CCS - Canadian Cardiovascular Society; EF — ejection fraction; MI - myocardial infarction; NYHA — New York Heart Association; PCI percutaneous coronary intervention; TIA — transient ischemic attack

\section{Discussion}

The MIDCAB surgery, along with all of its variations, is a well-established procedure in many cardiothoracic centers. Although PCI procedures have become increasingly effective over the last decades, both AHA/ACC and the European Society of Cardiology (ESC) and the European Association for Cardio-Thoracic Surgery (EACTS) guidelines underline the role of surgical revascularization in patients with stable angina and single vessel coronary heart disease with proximal LAD stenosis (IIA/B indication in AHA/ACC guidelines and IA indication in ESC/EACTS guidelines) [4, 9-10]. Furthermore, in a recent (2017) ACC report regarding the appropriate use of revascularization criteria, there was no discussion about possible changes in indications for surgical treatment in these cases [10].

The main purpose of the present research was to evaluate the short and long-term results of minimally invasive direct coronary artery bypass performed in patients operated on in the documented clinic in years between 1998 and 2009. Moreover, changes in the clinical profiles of the treated population happening over these years were analyzed.

The research showed that clinical characteristics of patients deteriorated, making treatment more challenging. Simultaneously, the frequency of complications was significantly reduced. This potential paradox can work as a confirmation of analysis which is well-described in the literature, a learning curve phenomenon, and meaning reduction in the number of complications due to excelling of surgical techniques and operator skills.

Research presented herein displays one of the biggest and longest observed groups of patients undergoing MIDCAB surgery. Mean follow-up amounted to $2132 \pm 1313$ days (Mdn: 1918.5, longest follow-up 4661 days). In the assessed time interval, frequency of MACCE was $10.8 \%$, with a death rate of $2.7 \%$ and a need for revascularization of $7 \%$. Bearing in mind the evaluated cumulative survival rate and event free survival of $96.9 \%$ and $85.3 \%$, respectively, indicates an optimal therapeutic effect.

As mentioned before, there are few studies regarding long-term observation after MIDCAB surgery. Holzhey et al. [11] reported long-term 
follow-up of more than 1300 patients with a death rate of $10.6 \%$ and cumulative MACCE $16.7 \%$ in 7-year observation. Hoffman et al. [12] presented his observation in an octogenarian group with a median survival rate of 6.7 years, which correlates well with the mean survival after CABG $[13,14]$ and with an age-adjusted general population. This study clearly demonstrated that the method provides satisfactory long-term outcomes, regardless of patient age group.

Blazek et al. [15] compared sirolimus-eluting stenting with minimally invasive bypass surgery for stenosis of the LAD. Although the groups were relatively small (65 patients each), the authors described excellent survival at median follow-up time of 7.3 years ( $17 \%$ death rate in the MIDCAB group) and a clear superiority of surgical revascularization in terms of repeat target vessel revascularization ( $20 \%$ vs. $1.5 \%, \mathrm{p}<0.01$ ). Similarly, Benedetto et al. [6] made a propensity score analysis of 303 MID$\mathrm{CAB}$ and 730 DES-PCI patients. They proved, that at 10 years DES-PCI was associated with a 2.19-fold increased risk of late death [6]. The survival rate after the MIDCAB procedure was $95.4 \pm 1.3$ and $94.8 \pm 1.3$ at 5 and at 10 years, respectively. Deppe et al. [16] performed meta-analysis of 2885 patients included in 12 studies. In all analyzed reports, patients were assigned to either PCI or MIDCAB group. The authors point out that MID$\mathrm{CAB}$ reduces repeat target vessel revascularization of the LAD and cumulative MACCE in long-term observation. It is noted that the incidence of MACCEs after PCI had almost doubled for the entire population after 6 months, which suggests that MIDCAB is an excellent strategy when longterm clinical outcome is required.

Finally, in the retrospective analysis performed, this study compared exclusive use of DES with MIDCAB in 463 consecutive patients. After adjustment at 5-year follow-up there were no differences in survival, MACCE free survival and MI survival between PCI and MIDCAB, respectively. However, there was a significantly higher freedom from repeated revascularization in patients who underwent MIDCAB [17].

It was considered essential to underline that this report is different from most studies as it refers to long-term follow up after minimally invasive surgical revascularization. The majority of large-group observations, including the Holzhey analysis of more than 1300 patients, which represented mainly or exclusively cases of direct vision LITA harvest. This makes the present observation quite unique and results seem to be even more valuable. The di- rect vision LITA harvesting may be associated with the risk of kinking, not to mention the necessity of costal resection or tough chest wall retraction. Furthermore, the endoscopy allows a complete dissection of the LITA from the subclavian artery to the sixth intercostal branches with transection of all collateral branches originating from LITA. This result is difficult to acquire under direct vision. In consequence, mastering the endoscopic LITA harvest ensures the avoidance of possible coronary steal syndrome $[18,19]$. Sabashnikov et al. [20] reported results of a team experienced in performing minimally invasive surgical revascularization in its various dimensions. They concluded that the endoscopic approach (EACAB) for LITA harvest is free from the disadvantages of longer operation duration observed in robotically assisted direct coronary artery bypass grafting (RADCAB) or higher incidence of angina and shorter freedom from MACEs observed in both MIDCAB and RADCAB groups [20]. This fact clearly demonstrates that both high quality anastomosis and perfect LITA harvest remain essential for satisfactory clinical outcome.

\section{Limitations of the study}

An inability to collect follow-up from approximately $25 \%$ of patients is a significant limitation of this research. However, the data obtained from the national registry database PESEL was crucial to the current study, as it allowed gathering of $100 \%$ follow-up regarding overall mortality. Additionally, the utilized method of data collection does not provide absolute certainty on whether renewed revascularization targeted the previously treated vessel or one of other coronary arteries. This information is vital for the proper evaluation of MIDCAB efficacy and can only be achieved through thorough evaluation of follow-up angiograms.

\section{Conclusions}

The presented short and long-term results confirm that MIDCAB (EACAB) is an efficacious alternative for patients requiring revascularization of the left anterior descending artery. The method is highly beneficial for the patient as it eliminates the use of cardiopulmonary bypass and avoids the sternotomy, which are both related to a number of complications widely described in the literature. The frequency of MACCE is low and acceptable, similar to a general population. However, it is to be remembered that the proper selection of patients in terms of both surgical risk and anatomical set- 


\section{ting of presented coronary artery lesions remains essential.}

\section{Conflict of interest: None declared}

\section{References}

1. Amsterdam EA, Wenger NK, Brindis RG, et al. 2014 AHA/ ACC Guideline for the Management of Patients with Non-STElevation Acute Coronary Syndromes: a report of the American College of Cardiology/American Heart Association Task Force on Practice Guidelines. J Am Coll Cardiol. 2014; 64(24): e139-e228, doi: 10.1016/j.jacc.2014.09.017, indexed in Pubmed: 25260718.

2. Dieberg G, Smart NA, King N. Minimally invasive cardiac surgery: A systematic review and meta-analysis. Int J Cardiol. 2016; 223: 554-560, doi:10.1016/j.ijcard.2016.08.227, indexed in Pubmed: 27557486.

3. Cisowski M. Miniinwazyjne pomostowanie gałęzi międzykomorowej przedniej lewej tętnicy wieńcowej $z$ wykorzystaniem techniki wideoskopowej. Rozprawa habilitacyjna. SUM, Katowice. 2004.

4. Windecker S, Kolh P, Alfonso F, et al. 2014 ESC/EACTS Guidelines on myocardial revascularization: The Task Force on Myocardial Revascularization of the European Society of Cardiology (ESC) and the European Association for Cardio-Thoracic Surgery (EACTS). Developed with the special contribution of the European Association of Percutaneous Cardiovascular Interventions (EAPCI). Eur Heart J. 2014; 35(37): 2541-2619, doi:10.1093/ eurheartj/ehu278, indexed in Pubmed: 25173339.

5. Wang XW, Qu C, Huang C, et al. Minimally invasive direct coronary bypass compared with percutaneous coronary intervention for left anterior descending artery disease: a meta-analysis. J Cardiothorac Surg. 2016; 11(1): 125, doi: 10.1186/s13019-0160512-1, indexed in Pubmed: 27491539.

6. Benedetto U, Raja SG, Soliman RFB, et al. Minimally invasive direct coronary artery bypass improves late survival compared with drug-eluting stents in isolated proximal left anterior descending artery disease: a 10-year follow-up, single-center, propensity score analysis. J Thorac Cardiovasc Surg. 2014; 148(4): 1316-1322, doi: 10.1016/j.jtcvs.2013.12.062, indexed in Pubmed: 24521955.

7. Yang M, Xiao LB, Gao ZS, et al. Clinical effect and prognosis of off-pump minimally invasive direct coronary artery bypass. Med Sci Monit. 2017; 23: 1123-1128, indexed in Pubmed: 28257412.

8. Hong SJ, Lim DS, Seo HS, et al. Percutaneous coronary intervention with drug-eluting stent implantation vs. minimally invasive direct coronary artery bypass (MIDCAB) in patients with left anterior descending coronary artery stenosis. Catheter Cardiovasc Interv. 2005; 64(1): 75-81, doi:10.1002/ccd.20238, indexed in Pubmed: 15619278.

9. Fihn S, Gardin J, Abrams J, et al. 2012 ACCF/AHA/ACP/AATS/ PCNA/SCAI/STS Guideline for the Diagnosis and Management of Patients With Stable Ischemic Heart Disease. J Am Coll Cardiol. 2012; 60(24): e44-e164, doi: 10.1016/j.jacc.2012.07.013.
10. Patel MR, Calhoon JH, Dehmer GJ, et al. ACC/AATS/AHA/ ASE/ASNC/SCAI/SCCT/STS 2017 Appropriate Use Criteria for Coronary Revascularization in Patients With Stable Ischemic Heart Disease: A Report of the American College of Cardiology Appropriate Use Criteria Task Force, American Association for Thoracic Surgery, American Heart Association, American Society of Echocardiography, American Society of Nuclear Cardiology, Society for Cardiovascular Angiography and Interventions, Society of Cardiovascular Computed Tomography, and Society of Thoracic Surgeons. J Am Coll Cardiol. 2017; 69(17): 2212-2241, doi: 10.1016/j.jacc.2017.02.001, indexed in Pubmed: 28291663.

11. Holzhey DM, Jacobs S, Mochalski M, et al. Seven-year follow-up after minimally invasive direct coronary artery bypass: experience with more than 1300 patients. Ann Thorac Surg. 2007; 83(1): 108-114, doi: 10.1016/j.athoracsur.2006.08.029, indexed in Pubmed: 17184640.

12. Hoffmann G, Friedrich C, Barrabas M, et al. Short- and longterm follow-up after minimally invasive direct coronary artery bypass in octogenarians. Interact Cardiovasc Thorac Surg. 2016; 23(3): 377-382, doi: 10.1093/icvts/ivw149, indexed in Pubmed: 27209534 .

13. Kozower BD, Moon MR, Barner HB, et al. Impact of complete revascularization on long-term survival after coronary artery bypass grafting in octogenarians. Ann Thorac Surg. 2005; 80(1): 112-116, doi: 10.1016/j.athoracsur.2005.02.017, indexed in Pubmed: 15975351.

14. Graham MM, Norris CM, Galbraith PD, et al. APPROACH Investigators. Quality of life after coronary revascularization in the elderly. Eur Heart J. 2006; 27(14): 1690-1698, doi: 10.1093/ eurheartj/eh1038, indexed in Pubmed: 16717072.

15. Blazek S, Rossbach C, Borger MA, et al. Comparison of sirolimuseluting stenting with minimally invasive bypass surgery for stenosis of the left anterior descending coronary artery: 7-year follow-up of a randomized trial. JACC Cardiovasc Interv. 2015; 8(1 Pt A): 30-38, doi:10.1016/j.jcin.2014.08.006, indexed in Pubmed: 25499302.

16. Deppe AC, Liakopoulos OJ, Kuhn EW, et al. Minimally invasive direct coronary bypass grafting versus percutaneous coronary intervention for single-vessel disease: a meta-analysis of 2885 patients. Eur J Cardiothorac Surg. 2015; 47(3): 397-406; discussion 406, doi: 10.1093/ejcts/ezu285, indexed in Pubmed: 25100715.

17. Buszman PP, Krol M, Cisowski M, et al. DES vs MIDCAB for proximal LAD disease: long term registry results. J Am Coll Cardiol. 2011; 58: B53.

18. Nataf P, Al-Attar N, Ramadan R, et al. Thoracoscopic IMA takedown. J Card Surg. 2000; 15(4): 278-282, indexed in Pubmed: 11758064.

19. Nataf P, Lima L, Regan M, et al. Minimally invasive coronary surgery with thoracoscopic internal mammary artery dissection: surgical technique. J Card Surg. 1996; 11(4): 288-292, indexed in Pubmed: 8902643.

20. Sabashnikov A, Patil NP, Weymann A, et al. Outcomes after different non-sternotomy approaches to left single-vessel revascularization: a comparative study with up to 10-year follow-up. Eur J Cardiothorac Surg. 2014; 46(4): e48-e55, doi: 10.1093/ejcts/ ezu287, indexed in Pubmed:25064052. 